\title{
Hydrodynamik von Kerndipolschwingungen ${ }^{1}$
}

\author{
Von Helmut Steinwedel und J. Hans D. Jensen \\ Aus dem Institut für theoretische Physik der Universität Heidelberg \\ (Z. Naturforschg. 5 a, 413-420 [1950]; eingegangen am 3. Juli 1950)
}

\begin{abstract}
Die kürzlich gefundenen Resonanzen für $(\gamma ; x)$-Prozesse werden entsprechend einem Vorschlag von G o ld h a b e r und T e l l e r als Dipolschwingungen der „Protonenflüssigkeit“ gegen die „Neutronenflüssigkeit“ im Kern gedeutet. Die frequenzbestimmenden Hookeschen Kräfte lassen sich ohne willkürliche Parameter entnehmen aus einer sinngemäßen Interpretation der halbempirischen Formel für die Bindungsenergien stabiler Kerne. Es ergibt sich eine ungewohnte Form der Hydrodynamik, die sich jedoch bequem aus dem Hamiltonschen Prinzip herleiten und integrieren läßt. In der einfachsten Näherung, in der Änderungen der Gesamtdichte, Kopplung mit Oberflächenschwingungen sowie der Einfluß der Störung der Coulombenergie unberücksichtigt bleiben, ergeben sich die Resonanzfrequenzen gegenüber den experimentellen um etwa $30 \%$ zu klein, zeigen jedoch den experimentell vorliegenden Gang mit der Massenzahl; höhere Näherungen verbessern die Übereinstimmung. Die Wirkungsquerschnitte für Absorption und Resonanzstreuung der $\gamma$-Strahlen werden diskutiert.
\end{abstract}

$\mathrm{D}$ ie neuerlich gefundenen ${ }^{2,3,4}$ Resonanzen bei $\gamma$ Energien um $20 \mathrm{MeV}$ für verschiedene $(\gamma ; \mathrm{x})$ Prozesse $(x=p$, bzw. $n$, bzw. $2 \mathrm{n}$, bzw. fission) wurden von Goldhaber und Teller ${ }^{5}$ als Eigenfrequenzen einer Kerndipolschwingung gedeutet, wobei sich der Protonenschwerpunkt im Kern gegen den Neutronenschwerpunkt bewegt, in der Art der Bewegung der Ionen im Kristall bei den ultraroten Reststrahlfrequenzen. Insbesondere der Befund von $\mathrm{B}$ a ldw in ${ }^{4}$, daß beim ${ }^{19} \mathrm{~F}$ die Resonanzstelle für den $(\gamma ; \mathrm{n})$ - und für den $(\gamma ; 2 \mathrm{n})$-Prozeß bei derselben Frequenz $\hbar \omega=$ etwa $30 \mathrm{MeV}$ liegt, läßt die Goldhaber-Tellersche Deutung der Resonanzen als Eigenfrequenz einer Kollektivbewegung der Ladungsträger im Kern sehr plausibel erscheinen. Die Nucleonenemission ist dann so zu verstehen, daß durch innere Reibung die angeregte geordnete Eigenschwingung in ungeordnete Bewegung dissipiert wird, und die damit verbundene Aufheizung des Kerns im Bohrschen Bilde des „Compound“-Kerns zum Nucleonenabdampfen führt; wegen einer genaueren Diskussion dieser Vorstellung vgl. 5 .

Die quantitative Diskussion stützen Goldhaber und Teller auf ein sehr vereinfachtes Modell, bei dem

1 Kurze Notiz, J. H. D. J e n s e n u. P. J e n s e n, Z. Naturforschg. 5a, 343 [1950]; vgl. auch Fußnote 5.

2 C. G. B a ldwi n u. G. S. K l a i ber, Physic. Rev. 71, 3 [1947]; 73, 1156 [1948]; M. L. P e a rlm a n u. G. Fried lände r, Physic. Rev. 74, 442 [1948]; J. L. L a w s o n u. M. L. P e a r l m a n, Physic. Rev. 74, 1190 [1948]; J. McElhin ney, A. O. H a u s e n, R. A. Bekker, R. B. D uffield u. B. C. Diven, Physic. Rev. 75, 542 [1949]; K. S t r a u c h, Physic. Rev. 78, 84 [1950]. die Neutronenkugel starr gegen die Protonenkugel schwingt. Hierbei sind jedoch die Hookeschen Kräfte, die die Frequenz bestimmen, nicht ohne (freilich plausible) willkürliche Parameter abzuschätzen ${ }^{5,6}$, und es schien uns deshalb reizvoll, ein anderes (physikalisch näherliegendes) Modell, das bei Goldhaber und Teller nur kurz erwähnt wird, genauer zu untersuchen, dem die nachstehenden Voraussetzungen zugrunde liegen. Es hat überdies den Vorteil, daß zur Berechnung der Eigenschwingungen keine willkürlichen Parameter erforderlich sind.

Voraussetzungen: 1. Die Gesamtdichte $\varrho_{0}$ (Zahl von Nucleonen pro $\mathrm{cm}^{3}$ ) ist konstant, $\varrho_{0}=\varrho_{\mathrm{p}}+\varrho_{\mathrm{n}}$ ( $\varrho_{\mathrm{p}}=$ Zahl von Protonen pro $\mathrm{cm}^{3}$, usw.); dies ist sicher eine zuverlässige Näherung, da die Kompressibilität der Kernmaterie äußerst gering ist; [Proportionalität des Kernradius mit $A^{1 / s}$, unabhängig von $\left.Z\right]^{7}$.

2. Die Kugelgestalt wird erhalten; d.h. wir sehen ab von einer Kopplung der Dipolschwingung mit

3 E. R. G a ert ner u. M. L. Y e a t e r, Physic. Rev. 76, 363 [1949]; 77, 714 [1950]; A. C. Helm holtz u. K. S t r a u ch, Physic. Rev. 78, 86 [1950]; R. D r es s e l, M. Goldh a b e r u. A. O. H a n s o n, Physic. Rev. 77, 754 [1950].

4 C. G. B a l d wi n, Physic. Rev. 76, 182 [1949] u. frdl. briefliche Mitteilung an P. J e n s e n.

5 M. Goldhaber u. G. T eller, Physic. Rev. 74, 1046 [1948].

6 Vgl. auch R. D. Pres ent, Physic. Rev. 77, 355 [1950].

7 Eine zuverlässige quantitative Abschätzung der Kernkompressibilität liegt noch nicht vor, vgl. E. F e e n b e r g, Rev. Mod. Physics 19, 239 [1947]; sicher ist sie jedoch so gering, daß sie für die nachfolgenden Betrachtungen irrelevant ist. 
Oberflächenschwingungen. Bei der Kleinheit der Amplituden, die sich unten ergibt, ist auch diese Näherung zu rechtfertigen, insbesondere weil nach der 1 . Voraussetzung die Dipolschwingung nicht mit einer Massenbewegung verbunden ist.

Die Dipolschwingung besteht dann in einer zeitlich veränderlichen lokalen Erhöhung der Protonendichte und entsprechender Verminderung der Neutronendichte (et vice versa):

$$
\varrho_{\mathrm{p}}=\varrho_{\mathrm{p}}{ }^{0}+\eta(\mathfrak{r}, t) \quad \text { und } \quad \varrho_{\mathrm{n}}=\varrho_{\mathrm{n}}{ }^{0}-\eta(\mathfrak{r}, t) .
$$

3. Die statischen Dichten $\varrho_{\mathrm{p}}{ }^{0}$ bzw. $\varrho_{\mathrm{n}}{ }^{0}$ setzen wir räumlich konstant:

$$
\varrho_{\mathrm{p}}^{0}=\frac{Z}{A} \varrho_{0} \quad \text { bzw. } \quad \varrho_{\mathrm{n}}{ }^{0}=\frac{N}{A} \varrho_{0}, \quad \text { mit } \frac{4 \pi}{3} r_{0}^{3} \varrho_{0}=1 \text {, }
$$

worin $r_{0}=e^{2} / 2 m c^{2}=1,42 \cdot 10^{-13} \mathrm{~cm}(m=$ Elektronenmasse, $c=$ Lichtgeschwindigkeit). Wegen des Anwachsens der Protonendichte gegen den Kernrand vgl. ${ }^{8}$; sein Einfluß auf die Eigenfrequenzen ist in einer nachfolgenden Arbeit ${ }^{9}$ berechnet. Er würde die Frequenzen erhöhen und einen etwas schwächeren Gang mit $A$ bewirken und dadurch die aus Abb. 1 ersichtliche Übereinstimmung mit den experimentellen Daten verbessern.

4. Die „rücktreibenden Kräfte“, die einer lokalen Erhöhung von $\varrho_{\mathrm{p}}$ bzw. $\eta$ entgegenwirken, lassen sich berechnen aus einer Energiedichte $\varepsilon\left(\varrho_{\mathrm{n}}-\varrho_{\mathrm{p}}\right)$, die nur vom lokalen Neutronenüberschuß abhängt. Diese läßt sich unmittelbar aus den empirischen Daten über die stabilen Kerne entnehmen. Der Ausdruck für die Bindungsenergie der Kerne ${ }^{10}$ enthält nämlich neben anderen, bei unseren Überlegungen als konstant anzusehenden Termen, einen Anteil für die Bindungsenergie pro Nucleon, der nur vom Neutronenüberschuß abhängt $E=K(N-Z)^{2} / A^{2}$, mit $K \cong 20 \mathrm{MeV}$. Wegen der kurzen Reichweite der Kernkräfte können wir diesen Term als Integral über eine lokale Energiedichte deuten, d.h. für die Energiedichte (Energie pro Volumeneinheit) können wir schreiben: $\varepsilon=\varrho_{0} E$. Weiter läßt sich gemäß (2) schreiben: $(N-Z) / A$ $=\left(\varrho_{\mathrm{n}}{ }^{0}-\varrho_{\mathrm{p}}{ }^{0}\right) / \varrho_{0}$, und dies in den Ausdruck für $E$ einsetzen; so erhält man die Energiedichte im statischen Gleichgewicht. Für den Fall lokaler Veränderungen der Protonendichte ergibt sich daraus durch Fortlassen des Index (0) der allgemeine Ausdruck für die

8 H. S te inwedel u. M. Danos, Physic. Rev., im Erscheinen.

${ }^{9}$ H. S t e inwed el u. M. D a n o s, S.-B. Heidelberger Akad. Wiss., im Manuskript.
Energiedichte:

$\varepsilon=K \frac{\left(\varrho_{\mathrm{n}}-\varrho_{\mathrm{p}}\right)^{2}}{\varrho_{0}}=K \frac{\left(\varrho_{0}-2 \varrho_{\mathrm{p}}\right)^{2}}{\varrho_{0}}=K \frac{\left(\varrho_{0}-2 \varrho_{\mathrm{p}}^{0}-2 \eta\right)^{2}}{\varrho_{0}}$.

H y d r o d y n a m i k. Mit diesen Ansätzen läßt sich konsequent die Hydrodynamik des Gegeneinanderströmens von „Neutronenflüssigkeit“ und „Protonenflüssigkeit“ unter Wahrung konstanter Gesamtdichte entwickeln. Die lokalen Strömungsgeschwindigkeiten $\mathfrak{v}_{\mathrm{p}}$ bzw. $\mathfrak{v}_{\mathrm{n}}$ der Protonen bzw. Neutronen sind, da keine Massenbewegungen auftreten, miteinander gekoppelt durch die Bedingung:

$$
\varrho_{\mathrm{p}} \mathfrak{y}_{\mathrm{p}}+\varrho_{\mathrm{n}} \mathfrak{y}_{\mathrm{n}}=0 .
$$

Man betrachtet zweckmäßig die „Relativgeschwindigkeit “ $\mathfrak{v}=\mathfrak{v}_{\mathrm{p}}-\mathfrak{v}_{\mathrm{n}}$ und führt die reduzierte Dichte ein:

$$
\varrho_{\text {red }}=\frac{\varrho_{\mathrm{p}} \varrho_{\mathrm{n}}}{\varrho_{0}} .
$$

Mit diesen Bezeichnungen ergeben sich, wie unten gezeigt wird, die „Eulerschen Gleichungen“:

$$
-\frac{\partial \varrho_{\mathrm{p}}}{\partial t}=\operatorname{div}\left(\varrho_{\mathrm{p}} \mathfrak{y}_{\mathrm{p}}\right)=\operatorname{div}\left(\varrho_{\mathrm{red}} \mathfrak{y}\right)
$$

als Kontinuitätsgleichung und, in der üblichen Beschränkung auf lineare Glieder in der Dichtestörung und Strömungsgeschwindigkeit, die Bewegungsgleichung:

$$
\begin{aligned}
& \mathrm{M} \frac{\partial \mathfrak{y}}{\partial t}=-\frac{\partial^{2} \varepsilon}{\partial \varrho_{\mathrm{p}}{ }^{2}} \operatorname{grad} \varrho_{\mathrm{p}}+e \mathcal{F}, \\
& \text { mit } \frac{\partial^{2} \varepsilon}{\partial \varrho_{\mathrm{p}}{ }^{2}}=\frac{8 \mathrm{~K}}{\varrho_{0}} \cong \frac{160 \mathrm{MeV}}{\varrho_{0}},
\end{aligned}
$$

worin $M$ die Nucleonenmasse und $\left[\begin{array}{r}\text { die } \\ \text { von den }\end{array}\right.$ Protonen erzeugte Feldstärke ist. Der Einfluß der von der Dichtestörung verursachten Feldstärke auf die Eigenschwingung ist gering ${ }^{11}$, wir wollen ihn im folgenden der Übersichtlichkeit halber fortlassen. Beim Einsetzen von (1) und (2) können wir (6) und (7), unter Beschränkung auf lineare Glieder in $\eta$ und $\mathfrak{v}$, umschreiben $\mathrm{zu}$ :

$-\dot{\eta}=\varrho^{0}$ red $\operatorname{div} \mathfrak{v}$, mit $\varrho^{0}{ }_{\text {red }}=\frac{\varrho_{\mathrm{p}}^{0} \varrho_{\mathrm{n}}^{0}}{\varrho_{0}}=\frac{Z N}{A^{2}} \varrho_{0}$

und

$$
M \dot{y}=-\frac{8 K}{\varrho_{0}} \operatorname{grad} \eta
$$

10 N. B o h r u. J. A. Wh e e le r, Physic. Rev. 56, 426 [1939]; vgl. auch 7 und die dort zitierten Arbeiten.

11 Vgl. die unter ${ }^{9}$ zitierte Arbeit, in der dieser Term konsequent berücksichtigt wird. 
Als Randbedingung haben wir zu fordern, daß am Kernrand die Radialkomponente der Protonenströmung, also auch die Radialkomponente von $\mathfrak{b}$ verschwindet; gemäß (9) muß also gelten:

$$
\frac{\partial \eta}{\partial r}=0 \quad \text { bei } \quad r=R=r_{0} A^{1 / 3}
$$

Elimination von $\mathfrak{v}$ aus (8) und (9) führt in der üblichen Weise zur Wellengleichung:

$$
\ddot{\eta}=u^{2} \operatorname{div} \operatorname{grad} \eta,
$$

worin $u$ als „Schallgeschwindigkeit“ zur Abkürzung eingeführt ist, gemäß

$$
u^{2}=\frac{\varrho^{0} \text { red }}{\varrho_{0}} \frac{8 K}{M}=\frac{Z N}{A^{2}} \frac{8 K}{M} .
$$

Es ist $u / c \cong 1 / \sqrt{23}$, die Fortpflanzungsgeschwindigkeit der Dichtestörung $\eta$ im Kern ist also gerade noch klein gegenüber der Lichtgeschwindigkeit. Da außerdem, wie sogleich gezeigt wird, die Eigenwellenlänge der tiefsten Dipolschwingung von der Größenordnung des Kerndurchmessers ist, folgt aus (12), daß die zur gleichen Frequenz gehörige elektromagnetische Wellenlänge gerade noch groß gegen die Kerndimensionen ist, so daß die Anregung der Schwingung durch $\gamma$ Strahlen noch in einfacher Weise zu berechnen ist. Für die Oberschwingungen müßte man freilich Retardierungseffekte berücksichtigen (vgl. ${ }^{5}$ und ${ }^{9}$ ).

Eigens chwingungen. Die Gl. (11) mit der Randbedingung (10) ist von Lord Rayle i g h ${ }^{12}$ ausführlich behandelt. Der Ansatz für Dipolschwingungen:

$$
\eta=e^{i \omega t} y(x) \cos \vartheta
$$

mit $x=r / \Lambda$ und $\Lambda=u / \omega$ ergibt für $\Lambda_{0}$, die ,durch $2 \pi$ dividierte Wellenlänge“ der untersten Eigenschwingung:

$R / \Lambda_{0}=2,08$, also $\Lambda_{0}=R / 2,08=r_{0} A^{1 / 3} / 2,08$.

Daraus berechnet sich die Eigenfrequenz $\omega_{0}$ gemäß $\omega_{0}=u / \Lambda_{0}=2,08 u / R$. Einsetzen von $u$ aus (12) liefert:

$$
\left.\hbar \omega_{0}=\sqrt{4 \frac{Z N}{A^{2}}} \frac{60}{\sqrt[3]{A}} \quad \text { (in } \mathrm{MeV}\right) .
$$

Abb. 1 gibt einen Vergleich mit den experimentellen Resonanzfrequenzen.

12 L ord Rayleigh, Theory of Sound, $2^{\text {nd }}$ ed., Chapt. 17, dtsch. Ausg. Vieweg-Verl. Braunschweig. 1880.
Die ohne willkürlichen Parameter berechneten Eigenfrequenzen sind bei den schwereren Elementen um etwa $30 \%$ zu klein. Die Abhängigkeit von $A$ wird befriedigend wiedergegeben; daß zu den leichtesten Kernen $(A<20)$ die theoretischen Frequenzen etwas stärker ansteigen als die experimentellen, ist nicht befremdend, da man nicht erwarten kann, daß bis zu so nucleonenarmen Kernen das Tröpfchenmodell eine physikalisch sinnvolle Näherung ist; die quantitative Übereinstimmung gerade beim $\mathrm{C}$ und Be möchten

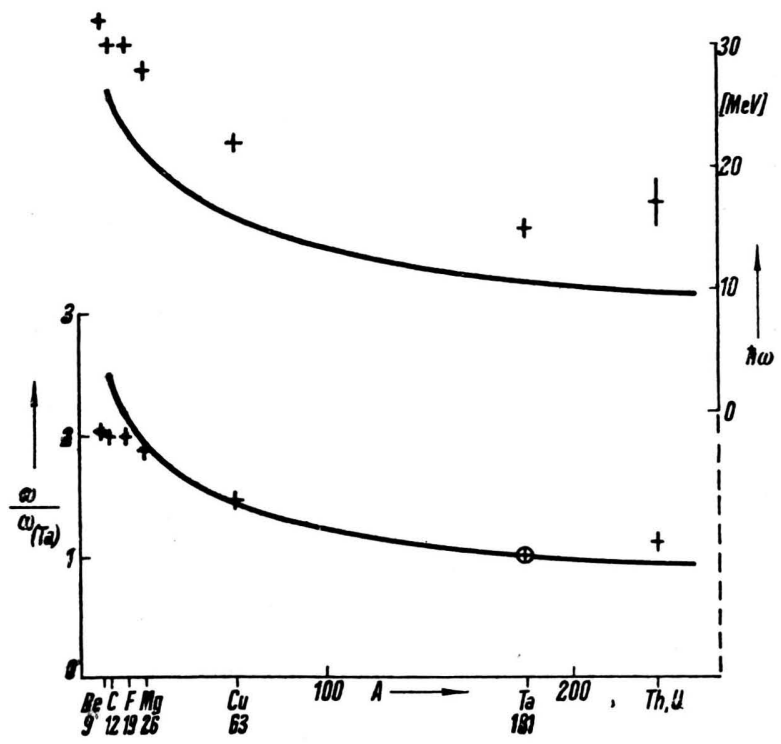

Abb. 1. Abszisse: Massenzahl. Ordinate: Resonanzfrequenzer für $\gamma ; \mathrm{x}$ ). Obere Kurve: Absolutwerte $\hbar \omega_{0}$ (in MeV). Kreuze: experimentelle Werte. Untere Kurve: Relativwerte, bezogen auf $\mathrm{Ta}^{181}: \omega_{0} / \omega_{0}(\mathrm{Ta})$.

wir als zufällig werten. Die im Vergleich mit $\mathrm{Ta}^{181}$ etwas reichlich hohe Lage der Resonanzstelle für $(\gamma$; fission) bei Th und U könnte durch eine Zunahme der Spaltungswahrscheinlichkeit mit der Anregungsenergie vorgetäuscht sein, außerdem ist aus dem oben nach Gl. (12) diskutierten Grunde gerade bei den schwersten Kernen der Einfluß der Oberschwingungen auf die Absorptionskurve schwer abzuschätzen.

Amplituden. Berechnet man in elementarer Weise die Maximalamplitude $\eta$ am Kernrand, die klassisch zur Anregung einer Schwingungsenergie vom Betrag $\hbar \omega_{0}$ gehört, so ergibt sich $\eta_{\max } / \varrho_{0} \cong 1,5 / A^{2 / 3}$. Also auch bei den leichten Kernen ist die Dichtestörung noch so klein, daß die Benutzung der linearen Hydrodynamik gerechtfertigt ist und wir den Strömungsvorgang im Kern nicht als "Stoßwelle“ zu behandeln brauchen. Immerhin ist $\eta_{\max } / \varrho_{0}$ doch schon 
so groß, daß die Abweichung von $30 \%$ gegenüber den experimentellen Frequenzen vielleicht in erster Linie dem Fehler in dieser Approximation zuzuschreiben ist.

Anregung der Eigenschwingung durch $\gamma$-Strahlen und Dämpfung (Wirkungsquerschnitte und Linienbreiten). Im „äußeren“ Strahlungsfeld der Frequenz $\omega$ ist die Bewegungsgleichung (7) bzw. (9) rechts durch ein Glied $e \mathfrak{E}_{\mathrm{s}} e^{i \omega t} \mathrm{zu}$ erweitern, wobei unter der Voraussetzung, daß die Strahlungswellenlänge groß gegen Kerndimensionen ist, $\mathfrak{\mho}_{\mathrm{s}}$ räumlich konstant angenommen werden kann; die Beeinflussung der Schwerpunktsbewegung durch das äußere Feld ist für uns nicht von Interesse (vgl. unten). Die Dissipation der geordneten Bewegung beschreiben wir sinngemäß $\beta^{5}$ durch ein zur Relativgeschwindigkeit proportionales Reibungsglied $-M \Gamma \mathfrak{v}$; die Viskositätskonstante $\Gamma$, für die es bislang kaum eine zuverlässige theoretische Abschätzung gibt, ist unmittelbar verknüpft mit der empirischen Linienbreite ${ }^{5}$, vgl. unten Gl. (17). Die so resultierende Bewegungsgleichung

$$
\dot{\mathfrak{y}}+\frac{8 K}{M \underline{o}_{0}} \operatorname{grad} \eta+\Gamma \mathfrak{v}=\frac{e}{M} \S_{\mathrm{s}} e^{i \omega t}
$$

zusammen mit der unveränderten Kontinuitätsgleichung (8) liefert als Lösung (Durchführung der Rechnung s. u.) bei Integration über das Kernvolumen ein oszillierendes Dipolmoment von der Stärke

$\mathfrak{p}=\Xi_{s} e^{i \omega t} \frac{N Z}{A^{2}} \frac{e^{2}}{M} \frac{1}{\left(R / A_{0}\right)^{2}-2} \frac{1}{\left(\omega^{2}-\omega_{0}^{2}\right)+i \Gamma \omega}$,

worin gemäß Gl. (14): $R / \Lambda_{0}=2,08$ ist; Gl. (17) gilt nur in der Umgebung der ersten Resonanzfrequenz, die kleinen Beiträge der höheren Resonanzstellen sind nicht mit angeschrieben, vgl. unten die vollständige Gl. (68). Der Phasenwinkel $\varphi$ zwischen erregender Feldstärke und Dipolmoment ist, wie in der Dispersionstheorie, gegeben durch

$\operatorname{tg} \varphi=\frac{\Gamma \omega}{\omega^{2}-\omega_{0}^{2}} \quad$ bzw. $\sin ^{2} \varphi=\frac{(\Gamma \omega)^{2}}{\left(\omega^{2}-\omega_{0}^{2}\right)^{2}+\left(\Gamma^{\prime} \omega\right)^{2}}$.

Aus dem Dipolmoment berechnet man in bekannter Weise die Ausstrahlung und aus $\overline{\left(\mathfrak{F}_{\mathrm{s}} \dot{\mathfrak{p}}\right)}$ die Absorption, vgl. unten Gl. (69) bis (74).

Der gesamte Wirkungsquerschnitt ergibt sich so zu

$$
\begin{gathered}
\sigma=\frac{\overline{\left(\Xi_{s} \dot{\mathfrak{p}}\right)}}{\bar{S}} \\
=8 \pi \frac{N Z}{A} \frac{m}{M} \frac{\hbar c}{e^{2}} \frac{1}{\left(R / \hat{\lambda}_{0}\right)^{2}-2} \frac{2 m c^{2}}{\hbar T}\left(\frac{e^{2}}{2 m c^{2}}\right)^{2} \sin ^{2} \varphi
\end{gathered}
$$

mit $\bar{S}=c / 8 \pi\left|\check{夭}_{\mathrm{s}}\right|^{2}=$ Mittelwert des Betrages des Poynting-Vektors. Für den Streuquerschnitt erhält man

$$
\sigma_{\text {Str. }}=\frac{\frac{2}{3} \frac{\omega^{4}}{c^{3}} \overline{\mathfrak{p}^{2}}}{\bar{S}}=\sigma \frac{\Gamma_{\mathrm{s}}}{\Gamma},
$$

worin $\Gamma_{\mathrm{s}}$ die Strahlungsbreite ist, in unserem Fall:

$$
\frac{\hbar \Gamma_{\mathrm{s}}}{2 m c^{2}}=\frac{4}{3} \frac{N Z}{A} \frac{m}{M} \frac{e^{2}}{\pi c} \frac{1}{\left(R / \Lambda_{0}\right)^{2}-2}\left(\frac{\hbar \omega}{2 m c^{2}}\right)^{2} .
$$

Für den integrierten Wirkungsquerschnitt folgt aus (19)

$$
\hbar \int_{0}^{\infty} \sigma d \omega
$$

$=4 \pi^{2} \frac{N Z}{A} \frac{m}{M} \frac{\hbar c}{e^{2}} \frac{1}{\left(R / \Lambda_{0}\right)^{2}-2}\left(\frac{e^{2}}{2 m c^{2}}\right)^{2} 2 m c^{2}$.

(22) stimmt bis auf den Faktor $\frac{1}{\left(R / \Lambda_{0}\right)^{2}-2}=\frac{1}{2,33}$ $=0,429$ mit dem von Goldhaber und Teller angegebenen Wert überein. Numerisch erhalten wir aus (19), (21) und (22), wenn wir $N Z / A^{2} \cong 1 / 4$ setzen:

$$
\begin{aligned}
\sigma & =4,12 \frac{2 m c^{2}}{\hbar \Gamma} A \sin ^{2} \varphi \cdot 10^{-27} \mathrm{~cm}^{2}, \\
\frac{\hbar \Gamma_{\mathrm{s}}}{2 m c^{2}} & =0,57\left(\frac{\hbar \omega}{2 m c^{2}}\right)^{2} A \cdot 10^{-6}, \\
\hbar \int_{0}^{\infty} \sigma d \omega & =6,5 \mathrm{~A} \cdot 10^{-27} \mathrm{~cm}^{2} \mathrm{MeV} .
\end{aligned}
$$

Nach den Experimenten ist $\hbar \Gamma \sim 2 \mathrm{MeV}$, somit ergeben sich für die Linienmitte die Werte

$$
\begin{gathered}
\sigma \sim 2 A \cdot 10^{-27} \mathrm{~cm}^{2}, \\
\frac{\pi \Gamma_{s}}{2 m c^{2}} \sim 2 A^{1 / 3} \cdot 10^{-3} .
\end{gathered}
$$

Die Wirkungsquerschnitte sind demnach so klein, daß sie für die Gesamtabsorption neben der ComptonStreuung und Paarerzeugung kaum eine Rolle spielen. Der Gesamtquerschnitt beträgt bestenfalls einige Prozent des Querschnitts für Compton-Streuung + Paarerzeugung, während der Streuquerschnitt, der 
noch um einen Faktor $10^{-3}-10^{-2}$ kleiner als der Absorptionsquerschnitt ist, allenfalls bei genauer Analyse (Energie, Winkelverteilung) der Streustrahlung nachweisbar wäre.

\section{Durchführung der Rechnungen}

Die Lagrange-Funktion und die Bewegungsgleichungen. Die Bewegungsgleichungen lassen sich am bequemsten aus dem Hamiltonschen Prinzip

$$
\delta I=\delta \int L d t=0
$$

ableiten. Die Lagrange-Funktion $L$ ist bei konservativen Kräften definiert als

$$
L=T-U,
$$

wobei $T$ die gesamte kinetische, $U$ die gesamte potentielle Energie ist. In unserem Falle ist die kinetische Energie

$$
T=\int\left\{\frac{M}{2} \varrho_{\mathrm{p}} \mathfrak{y}_{\mathrm{p}}^{2}+\frac{M}{2} \varrho_{\mathrm{n}} \mathfrak{y}_{\mathrm{n}}{ }^{2}\right\} d \tau .
$$

Wenn wir wie oben, Gln. (4) und (5), die Relativgeschwindigkeit $\mathfrak{v}=\mathfrak{v}_{\mathrm{p}}-\mathfrak{v}_{\mathrm{n}}$, und die reduzierte Dichte $\varrho_{\text {red }}=\varrho_{\mathrm{p}} \varrho_{\mathrm{n}} / \varrho_{0}$ einführen, und außerdem setzen:

$$
\mathfrak{B}=\frac{1}{\varrho_{0}}\left(\varrho_{\mathrm{p}} \mathfrak{y}_{\mathrm{p}}+\varrho_{\mathrm{n}} \mathfrak{y}_{\mathrm{n}}\right),
$$

so ergibt sich aus (26)

$$
T=\int\left\{\frac{M}{2} \varrho_{0} \mathfrak{D}^{2}+\frac{M}{2} \varrho_{\text {red }} \mathfrak{y}^{2}\right\} d \tau .
$$

Für die potentielle Energie erhalten wir entsprechend dem vorhergehenden Abschnitt, vgl. Gl. (3),

$$
U=\int\left\{K \frac{\left(\varrho_{\mathrm{p}}-\varrho_{\mathrm{n}}\right)^{2}}{\varrho_{0}}+e \varrho_{\mathrm{p}} \psi_{\mathrm{s}}\right\} d \tau
$$

wobei $\psi_{\mathrm{s}}$ das elektrostatische Potential des Strahlungsfeldes ist ${ }^{13}$. Zusätzlich zu (24) muß man noch das Bestehen der Kontinuitätsgleichungen fordern:

$$
\begin{aligned}
\frac{\partial \varrho_{\mathrm{p}}}{\partial t} & =-\operatorname{div} \varrho_{\mathrm{p}} \mathfrak{y}_{\mathrm{p}}, \\
\frac{\partial \varrho_{\mathrm{n}}}{\partial t} & =-\operatorname{div} \varrho_{\mathrm{n}} \mathfrak{y}_{\mathrm{n}}, \\
\quad( & \left.=-\frac{\partial \varrho_{\mathrm{p}}}{\partial t} \text { wegen } \varrho_{\mathrm{p}}+\varrho_{\mathrm{n}}=\varrho_{0}=\text { const }\right) .
\end{aligned}
$$

13 Die das Vektorpotential $\mathfrak{A}_{\mathrm{s}}$ enthaltenden Terme sind, da wir uns nur für den Fall kleiner Strömungsgeschwindigkeiten interessieren und diese Terme den Faktor $\mathfrak{b} / c$ enthalten, nicht berücksichtigt worden.
Aus (27) und (30) folgt zunächst

und ferner

$$
\operatorname{div} \mathfrak{B}=0
$$

$$
\frac{\partial \varrho_{\mathrm{p}}}{\partial t}=-\operatorname{div} \varrho_{\mathrm{red}} \mathfrak{v}-\operatorname{div} \varrho_{\mathrm{p}} \mathfrak{B},
$$

oder wegen (31)

$$
\frac{\partial \varrho_{\mathrm{p}}}{\partial t}+\left(\mathfrak{B}, \operatorname{grad} \varrho_{\mathrm{p}}\right)=-\operatorname{div} \varrho_{\text {red }} \mathfrak{y} .
$$

(31) ist nur eine andere Schreibweise für die vorausgesetzte räumliche und zeitliche Konstanz von $\varrho_{0}=\varrho_{\mathrm{p}}+\varrho_{\mathrm{n}}$. $\mathfrak{B}$ ist also, da wir auch rot $\mathfrak{B} \equiv 0$ setzen können, die (räumlich konstante) Geschwindigkeit des ganzen Kerns. In (33) bedeutet die linke Seite $\frac{\partial \varrho_{p}}{\partial t}$, gemessen in einem mit dem Kern mitbewegten Koordinatensystem. In diesem ist $\mathfrak{V}=0$ und (33) reduziert sich auf

$$
\frac{\partial \varrho_{\mathrm{p}}}{\partial t}=-\operatorname{div} \varrho_{\mathrm{red}} \mathfrak{v} .
$$

Bei der Variation (24) berücksichtigen wir $\varrho_{\mathrm{p}}+\varrho_{\mathrm{n}}=\varrho_{0}$ explizit und führen die Kontinuitätsgleichung (34) als Nebenbedingung mit Lagrangeschem Multiplikator $\lambda$ ein. Ferner setzen wir auch $\mathfrak{v}$ als wirbelfrei voraus und führen ein Geschwindigkeitspotential ein, gemäß

$$
\mathfrak{v}=-\operatorname{grad} \varphi .
$$

Unser Variationsproblem lautet dann

$$
\begin{gathered}
\delta \int d t \int d \tau\left\{\frac{M}{2} \varrho_{0} \mathfrak{B}^{2}+\frac{M}{2} \varrho_{\mathrm{red}}(\operatorname{grad} \varphi)^{2}-K \frac{\left(\varrho_{\mathrm{p}}-\varrho_{\mathrm{n}}\right)^{2}}{\varrho_{0}}\right. \\
\left.-e \varrho_{\mathrm{p}} \psi_{\mathrm{s}}+\lambda\left(\frac{\partial \varrho_{\mathrm{p}}}{\partial t}-\operatorname{div} \varrho_{\mathrm{red}} \operatorname{grad} \varphi\right)\right\}=0 .
\end{gathered}
$$

Die Variation von $\varphi$ und $\varrho_{\mathrm{p}}$ liefert in bekannter Weise

$$
M \operatorname{div} \varrho_{\text {red }} \operatorname{grad} \varphi+\operatorname{div} \varrho_{\text {red }} \operatorname{grad} \lambda=0,
$$

$$
\begin{gathered}
\frac{M}{2} \frac{\left(\varrho_{\mathrm{n}}-\varrho_{\mathrm{p}}\right)}{\varrho_{0}}(\operatorname{grad} \varphi)^{2}-4 K \frac{\varrho_{\mathrm{p}}-\varrho_{\mathrm{n}}}{\varrho_{0}}-e \psi_{\mathrm{s}} \\
-\frac{\partial \lambda}{\partial t}+\frac{\varrho_{\mathrm{n}}-\varrho_{\mathrm{p}}}{\varrho_{0}}(\operatorname{grad} \varphi, \operatorname{grad} \lambda)=0 .
\end{gathered}
$$

Aus (37) folgt $\lambda=-M \varphi$; damit wird aus (38)

$$
\begin{aligned}
M \frac{\partial \varphi}{\partial t}+\frac{M}{2} \frac{\varrho_{\mathrm{p}}-\varrho_{\mathrm{n}}}{\varrho_{0}} & (\operatorname{grad} \varphi)^{2} \\
& -4 K \frac{\varrho_{\mathrm{p}}-\varrho_{\mathrm{n}}}{\varrho_{0}}-e \psi_{\mathrm{s}}=0 .
\end{aligned}
$$


Eine elegantere Form des Hamiltonschen Prinzips erhält man, wenn man $\lambda=-M \varphi$ nachträglich in (36) berücksichtigt. Nach partieller Integration des mit $\lambda$ multiplizierten Terms sowie unter Berücksichtigung der räumlichen Konstanz von $\mathfrak{B}$ ergibt sich statt (36):

$$
\delta \int d t\left\{\int d \tau M \varrho_{\mathrm{p}} \frac{\partial \varphi}{\partial t}-H\right\}=0,
$$

wobei $H$ die Gesamtenergie ist:

$H=\frac{A M}{2} \mathfrak{B}^{2}$

$+\int d \tau\left\{\frac{M}{2} \varrho_{\text {red }}(\operatorname{grad} \varphi)^{2}+K \frac{\left(\varrho_{\mathrm{p}}-\varrho_{\mathrm{n}}\right)^{2}}{\varrho_{0}}+e \varrho_{\mathrm{p}} \psi_{\mathrm{s}}\right\}$.

(40) liefert bei Variation von $\varphi$ und $\varrho_{\mathrm{p}}$ neben der Bewegungsgleichung (39) auch gleichzeitig die Kontinuitätsgleichung (34). Bei Variation des Schwerpunkts-Ortsvektors $\mathfrak{R}(\dot{\Re}=\mathfrak{B})$ ergibt sich aus (40) wegen

$$
\delta \psi_{\mathbf{s}}=\delta \mathfrak{R} \operatorname{grad} \psi_{\mathbf{s}}
$$

die Bewegungsgleichung für den Schwerpunkt:

$$
A M \frac{d^{2} \Re}{d t^{2}}=-\int \varrho_{\mathrm{p}} \operatorname{grad} \psi_{\mathrm{s}} d \tau
$$

Will man aus dem Variationsprinzip (40) die vollständige Bewegungsgleichung (16) einschließlich Dämpfungsterm erhalten, so muß man auf der linken Seite von (40) noch einen Term hinzufügen, der die von den (nichtkonservativen) Reibungskräften geleistete virtuelle Arbeit $\delta A$ enthält ${ }^{14}$ :

$$
\int d t \delta A=\int d t \int d \tau M \Gamma \varrho_{\text {red }}(\operatorname{grad} \varphi, \delta \mathfrak{r}) .
$$

$\delta \mathfrak{x}$ ist dabei die virtuelle relative Verrückung der Protonengegen die Neutronenflüssigkeit. Partielle Integration liefert wegen $\delta \varrho_{\mathrm{p}}=-\operatorname{div} \varrho_{\text {red }} \delta \mathfrak{r}$

$$
\int d t \delta A=\int d t \int d \tau M \Gamma \varphi \delta \varrho_{\mathrm{p}} .
$$

Berücksichtigung dieses Terms in (40) liefert statt (39), wenn wir uns nunmehr auf lineare Glieder beschränken:

$$
M \frac{\partial \varphi}{\partial t}-4 K \frac{\varrho_{\mathrm{p}}-\varrho_{\mathrm{n}}}{\varrho_{0}}-e \psi_{\mathrm{s}}+M \Gamma \varphi=0 .
$$

Gradientenbildung zeigt sofort die Identität mit (16).

Die Eigens chwingungen. Setzen wir zunächst $\psi_{\mathrm{s}} \equiv 0$, so ergeben sich aus (34) und (46) durch Elimination von entweder $\varphi$ oder $\varrho_{\mathrm{p}}$ unter Berücksichtigung der Substitution (1) und nach Streichung nichtlinearer Glieder in $\varphi$ und $\eta$ die beiden äquivalen-

14 Da die Reibungskraft nicht konservativ ist, kann man diesen Term nicht gleich in der Lagrange-Funktion (25), sondern erst bei der Variation (40) berücksichtigen.

15 Vgl. oben vor Gl. (10). ten Differentialgleichungen [vgl. auch (11)]:

$$
\frac{\partial^{2} \eta}{\partial t^{2}}+\Gamma \frac{\partial \eta}{\partial t}-u^{2} \Delta \eta=0
$$

mit der Randbedingung ${ }^{15}\left(\frac{\partial \eta}{\partial r}\right)_{r=R}=0 ;$ resp:

$$
\frac{\partial^{2} \varphi}{\partial t^{2}}+\Gamma \frac{\partial \varphi}{\partial t}-u^{2} \Delta \varphi=0
$$

mit der Randbedingung $\left(\frac{\partial \varphi}{\partial r}\right)_{r=R}=0$,

wobei wie oben $u^{2}=\frac{N Z}{A^{2}} \frac{8 K}{M}$.

Setzen wir $\eta=\eta_{0}(\mathfrak{r}) e^{i \omega t}$, so ergibt sich für $\eta_{0}$ die Differentialgleichung

$\Delta \eta_{0}+\frac{1}{\lambda^{2}} \eta_{0}=0, \quad$ Randbedingung $\left(\frac{\partial \eta_{0}}{\partial r}\right)_{r=R}=0$, mit

$$
\lambda^{2}=\frac{u^{2}}{\omega^{2}-i \Gamma \omega} .
$$

Mit Rücksicht auf das Dämpfungsglied und im Hinblick auf den in ${ }^{9}$ mitberücksichtigten Einfluß der Störung der Coulomb-Energie bringen wir eine kurze $\mathrm{Zu}$ sammenfassung der Lösung von Lord Rayleigh ${ }^{12}$. Wegen Anregung der Schwingungen durch homogene periodische Felder interessieren wir uns nur für Dipolschwingungen und machen den Ansatz:

$$
\eta=y(r) \cos \vartheta e^{i \omega t}
$$

Damit folgt aus (49):

$$
\frac{d}{d r}\left(r^{2} \frac{d y}{d r}\right)+\left(\frac{r^{2}}{\lambda^{2}}-2\right) y=0
$$

mit den Randbedingungen ${ }^{16}\left\{\begin{array}{l}\left(\frac{d y}{d r}\right)_{r=R}=0 \\ (y)_{r=0}=0 .\end{array}\right.$

(52) ist ein positiv definites ${ }^{\mathbf{1 7}}$ selbstadjungiertes Eigenwertproblem vom Sturm-Liouvilleschen Typ, es besitzt nur reelle, positive, einfache Eigenwerte $\lambda^{-2}$.

$16 \eta(0)$ darf natürlich nicht singulär werden. Für den hier betrachteten Fall der Dipolschwingungen folgt daraus zwangsläufig $y(0)=0$.

17 Für jede die Randbedingungen erfüllende Funktion ist, wie man leicht durch partielle Integration zeigt:

$$
\frac{1}{\lambda^{2}} \int_{0}^{\mathrm{R}} r^{2} y^{2} d r=2 \int_{0}^{\mathrm{R}} y^{2} d r+\int_{0}^{\mathrm{R}} r^{2} y^{\prime 2} d r, \quad \text { also } \quad \frac{1}{\hat{i}^{2}}>0 \text {. }
$$


Führen wir eine dimensionslose Variable $x$ ein:

$$
x=\frac{r}{\lambda} ; \quad X=\frac{R}{\lambda},
$$

so wird aus (52) (Striche bedeuten Differentiation nach $x$ ):

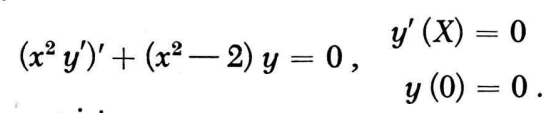

Die Lösung ist

$$
y=x^{-1 / 2} J_{3 / 2}(x)
$$

und die Randbedingung verlangt

$$
y^{\prime}(X)=X^{-3 / 2}\left(J_{3 / 2}(X)-X J_{5 / 2}(X)\right)=0
$$

oder, wenn man die Bessel-Funktionen in trigonometrischen Funktionen ausdrückt:

$$
\operatorname{tg} X=\frac{2 X}{2-X^{2}} .
$$

Diese Gleichung hat nach dem oben Gesagten die reellen Wurzeln ${ }^{12}$ :

$$
\begin{array}{cccccc}
n & 0 & 1 & 2 & 3 & \text { usw. } \\
X_{n} & \pm 2,08 & \pm 5,95 & \pm 9,20 & \pm 12,42 &
\end{array}
$$

asymptotisch: $X_{n}-X_{n-1}=\pi$.

Damit ergeben sich die Eigenfrequenzen zu

$\omega_{n}^{\prime}=\sqrt{\omega_{n}^{2}-\frac{\Gamma^{2}}{4}}+\frac{i \Gamma}{2}$ mit $\omega_{n}=\frac{X_{n}}{R} u$.

Erzwungene S chwingungen. Wenn $\psi_{\mathrm{s}} \neq 0$ ist, erhalten wir statt (47) die Differentialgleichung

$$
\frac{\partial^{2} \eta}{\partial t^{2}}+\Gamma \frac{\partial \eta}{\partial t}-u^{2} \Delta \eta=0
$$

mit der Randbedingung

$$
u^{2} \operatorname{grad}_{r} \eta=-\frac{e}{M} \frac{N Z}{A^{2}} \varrho_{0} \operatorname{grad}_{r} \psi_{s} \text { für } r=R,
$$

also (wegen $\Delta \psi_{\mathrm{s}}=0$ ) eine homogene Differentialgleichung mit inhomogener Randbedingung. Da $\operatorname{grad} \psi_{\mathrm{s}}$ räumlich konstant sein soll, werden nur Dipolschwingungen angeregt, und wir erhalten wie oben

$$
\eta=C \frac{J_{3 / 2}(x)}{x^{1 / 2}} \cos \vartheta e^{i \omega t} .
$$

Aus der Randbedingung folgt für $C$, wenn wir $\operatorname{grad} \psi_{\mathrm{s}}=\mathfrak{夭}_{\mathrm{s}} e^{i \omega t}$ setzen:

$$
C=u^{2} R \frac{N Z}{A^{2}} \varrho_{0} \frac{e}{M}\left|\S_{s}\right| \frac{X^{1 / 2}}{J_{3 / 2}(X)-X J_{5 / 2}(X)} .
$$

Wie oben erhalten wir eine äquivalente Differentialgleichung [das Analogon zu (48)], wenn wir aus den Bewegungsgleichungen $\eta$ eliminieren:

$$
\frac{\partial^{2} \varphi}{\partial t^{2}}+\Gamma \frac{\partial \varphi}{\partial t}-u^{2} \Delta \varphi=\frac{e}{M} \frac{\partial \psi_{s}}{\partial t}
$$

mit der Randbedingung

$$
\operatorname{grad}_{r} \varphi=0 \text { für } r=R,
$$

also im Gegensatz zu (59) eine inhomogene Gleichung mit homogener Randbedingung. Man findet leicht als Lösung

$$
\begin{aligned}
& \varphi=i \omega \frac{e}{M}\left|\Xi_{s}\right| \frac{R^{3}}{u^{2}} \frac{1}{X^{3}} \\
& \cdot\left\{x-\frac{X^{3 / 2}}{J_{3 / 2}(X)-X J_{5 / 2}(X)} \frac{J_{3 / 2}(x)}{x^{1 / 2}}\right\} \cos \vartheta e^{i \omega t} .
\end{aligned}
$$

Bei den Eigenfrequenzen (58) liegen die Nullstellen $X_{n}$ des "Resonanznenners“ $J_{3 / 2}(X)-X J_{5 / 2}(X)$. Für das Dipolmoment $\mathfrak{p}$ erhalten wir, wenn wir von der Schwerpunktsbewegung absehen,

$$
\begin{aligned}
& \mathfrak{p}=e \int \mathfrak{r} \eta(\mathfrak{r}) d \tau= \mathfrak{P} \frac{J_{5 / 2}(X)}{X\left(J_{3 / 2}(X)-X J_{5 / 2}(X)\right)} \\
& \text { mit } \mathfrak{P}=\frac{Z N}{A} \frac{R^{2}}{u^{2}} \frac{e^{2}}{M} \S_{s} e^{i \omega t} .
\end{aligned}
$$

Partialbruchzerlegung. (64) wird übersichtlicher, wenn man für $\mathfrak{p}(X)$ eine Partialbruchzerlegung ansetzt:

$$
\begin{gathered}
f(z)=\frac{J_{5 / 2}(z)}{z\left(J_{3 / 2}(z)-z J_{5 / 2}(z)\right)} \\
=-\frac{\left(3-z^{2}\right) \sin z-3 z \cos z}{z^{2}\left\{\left(2-z^{2}\right) \sin z-2 z \cos z\right\}} \\
=\sum_{n}\left(\frac{C_{n}}{z-z_{n}}+g_{n}(z)\right) .
\end{gathered}
$$

Die $z_{n}$ sind dabei die (nach dem oben Gesagten einfachen und reellen) Pole von $f(z)$. In unserem Falle gilt asymptotisch für große $z$ (abgesehen natürlich von der Umgebung der Pole $\left.z_{n}\right) f(z) \sim z^{-2}$. Wir erhalten somit nach der bekannten Methode von $\mathrm{Cauch}{ }^{18}$ :

$$
\begin{aligned}
& \frac{C_{n}}{z-z_{n}}+g_{n}(z)=-\frac{1}{2 \pi i} \oint_{\left(z_{n}\right)} \frac{f(\zeta)}{(\zeta-z)} d \zeta \\
& =\frac{1}{z_{n}} \frac{1}{z_{n}^{2}-2} \frac{1}{z_{n}-z}, \text { also } g_{n}(z) \equiv 0 .
\end{aligned}
$$

18 Vgl. z. B. Hurwitz-Courant, Funktionentheorie, Springer-Verlag Berlin 1929, S. $118 \mathrm{ff}$. 
Wie wir sahen, ist neben $+z_{n}$ auch stets $-z_{n}$ ein Pol von $f(z)$. Indem wir immer zwei entsprechende Terme (66) zusammenfassen, erhalten wir

$$
f(z)=2 \sum_{n} \frac{1}{z_{n}^{2}-2} \frac{1}{z_{n}^{2}-z^{2}} ;
$$

unter Berücksichtigung der Definition von $X$ ergibt sich somit

$$
\begin{aligned}
\mathfrak{p} & =2 \mathfrak{P} \sum_{n} \frac{1}{X_{n}{ }^{2}-2} \frac{1}{X_{n}{ }^{2}-X^{2}} \\
& =\frac{N Z}{A} \frac{e^{2}}{M} \S_{s} e^{i \omega t} \sum_{n} \frac{1}{X_{n}{ }^{2}-2} \frac{1}{\omega_{n}{ }^{2}-\omega^{2}+i \Gamma \omega} .
\end{aligned}
$$

Wirkungsquerschnitte. Da für die Oberschwingungen die Voraussetzung, daß die Lichtwellenlänge groß gegen Kerndimensionen ist, nicht mehr zutrifft, beschränken wir uns im folgenden auf die Umgebung der Grundfrequenz $\omega_{0}$. Unter Einführung der "Strahlungsbreite“ $\Gamma_{\mathrm{s}}$ gemäß Gl. (20),

$$
\frac{\hbar \Gamma_{s}}{2 m c^{2}}=\frac{\hbar}{2 m c^{2}} \frac{2}{3} \frac{N Z}{A} \frac{e^{2}}{M} \frac{\omega^{2}}{c^{3}} \frac{1}{X_{0}^{2}-2}
$$

und des in Gl. (18) angegebenen Phasenwinkels $\varphi$ zwischen erregender Feldstärke $\mathfrak{F}_{\mathrm{s}}$ und Dipolmoment $\mathfrak{p}$ erhalten wir die in (19) bis (23) angegebenen und diskutierten Wirkungsquerschnitte.

\title{
Austausch- und andere Zweizentrenintegrale mit $2 \mathrm{~s}$ - und 2 p-Funktionen
}

\author{
Von Hermann-Josef Kopineck \\ Aus dem Max-Planck-Institut für Physik, Göttingen \\ (Z. Naturforschg. 5 a, 420-431 [1950]; eingegangen am 21. Juni 1950)
}

$\mathrm{I}^{\mathrm{n}}$ m Rahmen ausführlicherer Untersuchungen der Iquantenmechanischen Theorie der chemischen Bindung erwies es sich als notwendig, ZweizentrenWechselwirkungsintegrale mit $2 \mathrm{~s}$ - und 2 p-Funktionen numerisch zu berechnen.

Im Anschluß an S u g i u r a ${ }^{1}$ - der als erster diese Integraltypen, und zwar für 1s-Elektronen berechnete - wurden von folgenden Autoren Zweizentrenintegrale mit $2 \mathrm{~s}$ - und $2 \mathrm{p}$-Funktionen angegeben:

Ros e ${ }^{2}$ tabelliert eine Anzahl von Hilfsintegralen, die zur Auswertung von Austauschintegralen, s. u. Gl. (4c), benutzt werden können.

D e l b r ü ck ${ }^{3}$ hat Wechselwirkungsintegrale zwischen $2 \mathrm{~s}^{2}$-Elektronen unter gewissen Vernachlässigungen ermittelt, so daß seine Berechnung des $\mathrm{Li}_{2}$ Moleküls noch ungenau ist. Einige Integrale zwischen $2 \mathrm{~s}^{2}$ - und $2 \mathrm{~s}, 2 \mathrm{p}$-Elektronen geben Bartlett und Furry ${ }^{4}$ an. Ihr Ergebnis ist in guter Úbereinstimmung mit unseren auf anderem Wege berechneten

1 Y. S u g i u r a, Z. Physik 45, 484 [1927].

2 N. R o s e n, Physic. Rev. 38, 255, 2099 [1931].

3 M. D e l b r ü c k, Ann. Physik 5, 36 [1930].

4 J. H. B a r t lett u. B. H. F u r y, Physic. Rev. 38, 1615 [1931]; 39, 209 [1932].

5 J. H. B a rt le t t, Physic. Rev. 37, 507 [1931].

6 W. E. B lei ch u. J. E. M a y er, J. chem. Physics 2, 252 [1934].

7 A. L. S k l a r, J. chem. Physics 6, 645 [1938]. A. L. Skla r u. R. H. Ly d d a n e, J. chem. Physics 7, 374 [1939].
Werten. Die zwischen $2 \mathrm{p}^{2}$-Elektronen auftretenden Integrale hat $\mathrm{B}$ a r t l e $\mathrm{t} \mathrm{t}^{5}$ als erster - aber noch mit Vernachlässigungen - berechnet. Einige numerische Werte von Wechselwirkungsintegralen zwischen $2 \mathrm{~s}^{2}$, $2 \mathrm{~s}, 2 \mathrm{p}$ - und $2 \mathrm{p}^{2}$-Elektronen finden sich außerdem bei Bleich und Mayer ${ }^{6}, \mathrm{Sklar}_{\mathrm{B}} \mathrm{bzw}$. Sklar und Lyddane ${ }^{7}$ und Wheatley und Linnett ${ }^{8}$. Sehr ausführlich und genau haben kürzlich $\mathrm{P}$ a r $\mathrm{r}$ und $\mathrm{Crawford}{ }^{9 a}$ sowie $\mathrm{Hirschfelder}$ und Linne $\mathrm{t}^{\mathrm{gb}}$ mehrere Integrale mit $2 \mathrm{p}$-Funktionen berechnet, die in guter Übereinstimmung mit unseren Werten sind. Alle diese Autoren benutzen bei der Auswertung der Integrale den von $\mathrm{R}$ os e $\mathrm{n}^{2}$ angegebenen Integrationsweg wie auch seine Hilfsintegraltabellen.

Kotany, Amemiya und Simose ${ }^{10}$ veröffentlichten 1938 eine sehr ausführliche Tabelle von Hilfsintegralen zur Berechnung von Austausch- und anderen Wechselwirkungsintegralen. Diese Tabellen, die sich u. a. durch die große Zahl der Werte des

8 P. J. Wheatley u. J. W. Linnett, Trans. Faraday Soc. 45, 897 [1949].

9a R. G. Parr u. B. L. C r a w f ord, J. chem. Physics 16, 1049 [1948].

9b J. O. Hirschfelder u. J. W. Linnett, J. chem. Physics 18, 130 [1950].

10 M. Kotany, A. Amemiya u. T. Simose, Proc. physico-math. Soc. Japan 20, Extra-Nr. 1 [1938]; 22, ExtraNr. 1 [1940]. 